\title{
Clinical and laboratory features that differentiate familial from sporadic focal segmental glomerulosclerosis in adolescents and adults
}

\author{
Maria Goretti Polito', Michelle Tiveron Passos' ${ }^{1}$, Danilo Euclides Fernandes', Gianna Mastroianni-Kirsztajn²* \\ 'Department of Medicine (Nephrology), Federal University of São Paulo, São Paulo-SP, Brazil \\ ${ }^{2}$ Associate Professor of Medicine, Department of Medicine (Nephrology), Federal University of São Paulo, São Paulo-SP, Brazil
}

\section{Article Info}

\section{Article Notes}

Received: October 27, 2020

Accepted: January 20, 2021

\section{*Correspondence:}

${ }^{*}$ Dr. Gianna Mastroianni-Kirsztajn, Associate Professor of Medicine, Department of Medicine (Nephrology), Federal University of São Paulo, São Paulo-SP, Brazil; Telephone No: 55-11-5904-1699; Fax No: 55-11-5904-1684; Email: gm.kirsztajn@unifesp.br

${ }^{\circ} 2021$ Mastroianni-Kirsztajn G. This article is distributed under the terms of the Creative Commons Attribution 4.0 International License.

\section{Keywords}

Focal segmental glomerulosclerosis (FSGS)

Familial FSGS

Sporadic FSGS

Proteinuria

Genetic mutation

Nephrotic syndrome

\section{ABSTRACT}

Background: Focal segmental glomerulosclerosis (FSGS) is an important cause of end-stage kidney disease in children and adults. Although most cases are sporadic (s), familial (f) presentation is also described. The purpose of the present study was to establish clinical and laboratory profiles of fFSGS vs. primary sFSGS, contributing to the distinguishing diagnosis in clinical practice and best management, in particular when mutation analysis is not available.

Methods: Demographic, clinical and laboratorial parameters were studied in 124 patients 12 years and older with FSGS, subdivided in $\mathrm{SFSGS}(\mathrm{n}=89)$ and fFSGS $(n=35)$.

Results: General and clinical features were similar, as well as serum creatinine at disease presentation. Proteinuria levels were more frequently $\geq 3 \mathrm{~g} /$ day in sFSGS $(63.8 \%)$ than in fFSGS $(44 \%, p=0.080)$, and serum albumin levels were $\leq 3.0 \mathrm{~g} / \mathrm{dL}$ in $45.8 \%$ and $20 \%$, respectively ( $\mathrm{c}=0.046$ ). The groups were statistically different regarding steroid resistance, corresponding to $60 \%$ in $\mathrm{sFSGS}$ and $100 \%$ in $\mathrm{fFSGS}(\mathrm{p}=0.001)$.

Conclusions: The studied groups were clinically similar, except that proteinuria tended to be higher (nephrotic range) and serum albumin was lower in SFSGS vs. FFSGS. In addition, all treated fFSGS patients were steroid resistant. At presentation it is important to characterize if the patient has fFSGS, that will contribute to further disease management, and disease history will be the first clue for such differential diagnosis.

\section{Introduction}

Focal segmental glomerulosclerosis (FSGS) represents a heterogenous group of diseases, which accounts for an important cause of end-stage kidney disease and manifests predominantly with nephrotic syndrome regardless of the age of onset. Although isolated mutations can cause sporadic forms of FSGS, some of those who present with FSGS carry a familial form that explains their FSGS pathogenesis $^{1-3}$.

More than 50 genes have shown pathogenic variants that have been associated with FSGS ${ }^{4}$. NPHS2 mutations were described in FSGS that happens from childhood to adulthood, usually presenting with high levels of proteinuria in both autosomal recessive familial FSGS (fFSGS) and sporadic FSGS (sFSGS) forms ${ }^{5-8}$. Other gene mutations are related to autosomal dominant FSGS, as in ACTN4, TRPC6, CD2AP 9,10 , and INF2 genes ${ }^{11}$. In fact, INF2 mutations are seen as the most common cause of autosomal dominant FSGS accounting for $9 \%$ of the cases, while ACTN4 e TRPC6 mutations are observed in 3 and $2 \%$ of them, respectively ${ }^{11}$. 
The primary forms of FSGS have been treated with steroids and immunosuppressive drugs, with variable frequency of remission, and recurrence after kidney transplantation is relatively common ${ }^{12,5}$. On the other hand, immunosuppressive treatment is not usually indicated in genetic cases of FSGS, thus this kind of approach remains a matter of concern while managing fFSGS. Nevertheless, calcineurin inhibitor cyclosporine ${ }^{13,14}$ or, less frequently, steroids ${ }^{15}$ can induce a partial remission depending on the genetic variants involved in each case.

Molecular diagnosis is not indispensable for treating this disease, but as pathogenic mutations are identified in FSGS, they can help to: (1) avoid adverse effects of steroids, (2) modify the intensity and duration of immunosuppressive therapies, (3) contribute to donor selection, and (4) eventually delay the progression of the disease and establish its prognosis. Genetic study also provides genotype-phenotype associations, which enables genetic counseling and may identify associated mutations ${ }^{4}$.

Few research centers and clinical analysis laboratories have the infrastructure and expertise to perform genetic analyses, whether by Sanger sequencing or next-generation sequencing. In addition, genetic investigation is expensive and remains routinely unfeasible in many countries.

Here, we describe clinical and laboratory features of fFSGS and sFSGS that could distinguish them in daily practice. This differentiation may help to guide the treatment approach, which may avoid unnecessary steroids and immunosuppressive drugs for most of those who have a family history of FSGS. Also, it can avoid doing renal biopsy in relatives and help in genetic counseling.

\section{Materials and Methods}

This is a retrospective and unique center cohort developed at Federal University of São Paulo, Brazil. This protocol was approved by the Ethical Committee on Research of the UNIFESP. Informed consent was obtained from all individual participants included in the study.

We included the patients with histological diagnosis of FSGS followed in the Section of Glomerular Diseases (Division of Nephrology) until 2016. The participants were divided into two groups, fFSGS (according to their familial history of chronic kidney disease or glomerular disease in close relatives as parents, brothers/sisters or siblings) and primary sFSGS.

We obtained demographic and clinical data from the patients' charts from which we collected laboratory results (serum creatinine, cholesterol, triglycerides, and albumin; and urinalysis and 24-hour proteinuria), familial history of chronic kidney disease or glomerular disease, blood pressure levels and treatment regimens. In case there were syndrome features those would also be described.
The response to steroid therapy was defined according to laboratory results as present (partial or complete remission) or not (maintenance of $24 \mathrm{~h}$-proteinuria above $3.0 \mathrm{~g} / 24 \mathrm{~h}$ ) after 16 weeks of treatment with oral prednisone $1 \mathrm{mg} / \mathrm{kg} /$ day (followed by a withdrawal schedule).

\section{Statistical analysis}

Data were analyzed by using SPSS statistics $\AA$, version 26. We present summarized results through relative and absolute frequencies (nominal variables) and mean and standard deviation (numerical variables). Inferential analyses were performed using Pearson's Chi-square, t-Student, and Mann-Whitney's tests, when appropriate. We set alpha $=0.05$ and $95 \%$ confidence interval for all the tests we used.

\section{Results}

We present clinical and laboratorial characteristics from 124 patients with FSGS, which are subdivided into two groups: primary sFSGS $(n=89)$ and fFSGS $(n=35)$. Both groups were statistically similar in gender $(42.9 \%$ vs. $52.8 \%$ males in fFSGS vs. $\mathrm{SFSGS}$, respectively, $\mathrm{p}=0.318$ ). The two groups were also similar with regards to their ethnicity ( $\mathrm{p}=0.454)$, but "white" (47.4\% and 59.6\%) and "neither black nor white" patients $(52.6 \%$ in fFSGS and $36.5 \%$ in sFSGS) predominated, while no "black" patients $(0 \%)$ were found in fFSGS vs. $3.8 \%$ in sFSGS.

The patients were diagnosed with FSGS when they were $34.1 \pm 10.5$ (fFSGS) and $32.3 \pm 12.7$ (sFSGS) years old, but they did not differ significantly $(\mathrm{p}=0.288)$.

The mean initial levels of serum creatinine $(\mathrm{mg} / \mathrm{dL})$ were $1.31 \pm 0.72$ (sFSGS) vs. $1.20 \pm 0.89$ (fFSGS) ( $\mathrm{p}=0.484$ ), the occurrence of hematuria was $72.4 \%$ (fFSGS) and $71.8 \%$ (sFSGS) ( $\mathrm{p}=0.949)$ and the mean absolute levels of 24-hour proteinuria $(\mathrm{g} / 24 \mathrm{~h})$ were $5.63 \pm 2.99$ (sFSGS) and 3.60 \pm 4.83 (fFSGS) ( $p=0.225)$. Higher levels of proteinuria $(\geq 3.0$ $\mathrm{g} /$ day) were more frequently observed in the $\mathrm{SFSGS}$ group $(63.8 \%)$, than in the fFSGS group, in which proteinuria < $3.0 \mathrm{~g} /$ day predominated $(56 \%)$. The $\chi^{2}$ test revealed a tendency to differentiating these groups $(\mathrm{p}=0.08)$.

Serum albumin $<3.0 \mathrm{~g} / \mathrm{dL}$ was more frequently observed in the sFSGS group (45.8\%), than in the fFSGS (20\%) one. On the other hand, the fFSGS group presented a higher prevalence of serum albumin $\geq 3.0 \mathrm{~g} / \mathrm{dL}(80 \%)$ $(\mathrm{p}=0.04)$.

Both groups were similar with regards to serum cholesterol $(\geq 200 \mathrm{mg} / \mathrm{dL} ; 47.4 \%$ in fFSGS and $66.7 \%$ in sFSGS, $p=0.140$ ), serum triglycerides $(\geq 150 \mathrm{mg} / \mathrm{dL} ; 47.1 \%$ in fFSGS and $54.0 \%$ in $\mathrm{sFSGS}, \mathrm{p}=0.621$ ) and hypertension ( $48.6 \%$ in fFSGS vs. $54.1 \%$ in $s F S G S, p=0.580$ ).

Steroids were given to some of the patients in both groups (14 in fFSGS and 62 in sFSGS). All the individuals 
Table 1: Clinical, laboratory and demographic data on the patients with familial and sporadic focal segmental glomerulosclerosis (FSGS)

\begin{tabular}{|c|c|c|c|c|}
\hline Parameter & & Familial FSGS & Sporadic FSGS & $p$-value \\
\hline \multicolumn{5}{|l|}{ Gender } \\
\hline Male & $\mathrm{n}(\%)$ & $15(42.9)$ & $47(52.8)$ & $0.318^{a}$ \\
\hline Female & & $20(57.1)$ & $42(47.2)$ & \\
\hline \multirow{4}{*}{ Age (years old) } & $\mathrm{n}$ & 31 & 88 & \multirow{4}{*}{$0.288^{b}$} \\
\hline & Mean & 34.1 & 32.3 & \\
\hline & Minimum-Maximum & $19-56$ & $14-66$ & \\
\hline & SD & 10.5 & 12.7 & \\
\hline \multirow{4}{*}{ Serum creatinine (mg/dL) } & $\mathrm{n}$ & 31 & 88 & \multirow{4}{*}{$0.484^{b}$} \\
\hline & Mean & 1.20 & 1.31 & \\
\hline & Minimum-Maximum & $0.36-4.00$ & $0.45-4.26$ & \\
\hline & SD & 0.69 & 0.72 & \\
\hline \multirow{4}{*}{ 24-hour proteinuria (g/24h) } & $\mathrm{n}$ & 29 & 78 & \multirow{4}{*}{$0.225^{\mathrm{c}}$} \\
\hline & Mean & 3.6 & 5.63 & \\
\hline & Minimum-Maximum & $0.49-13.23$ & $0.30-22.70$ & \\
\hline & SD & 4.83 & 2.99 & \\
\hline \multicolumn{5}{|l|}{ Hematuria (erythrocytes/mL) } \\
\hline$>8000$ & \multirow[t]{2}{*}{$\mathrm{n}(\%)$} & $21(72.8)$ & $56(71.8)$ & \multirow[t]{2}{*}{$0.949^{\mathrm{a}}$} \\
\hline$\leq 8000$ & & $8(27.6)$ & $22(28.2)$ & \\
\hline \multicolumn{5}{|l|}{ Serum albumin $(g / d L)$} \\
\hline$>3.0$ & $n(\%)$ & $16(80)$ & $26(54.2)$ & \multirow[t]{2}{*}{$0.046^{a}$} \\
\hline$\leq 3.0$ & & $4(20)$ & $22(45.8)$ & \\
\hline \multicolumn{5}{|l|}{ Serum cholesterol (mg/dL) } \\
\hline$\geq 200$ & $\mathrm{n}(\%)$ & $9(47.4)$ & $34(66.7)$ & \multirow[t]{2}{*}{$0.140^{\mathrm{a}}$} \\
\hline$<200$ & & $10(52.6)$ & $17(33.3)$ & \\
\hline \multicolumn{5}{|l|}{ Serum triglycerides $(\mathrm{mg} / \mathrm{dL})$} \\
\hline$\geq 150$ & $\mathrm{n}(\%)$ & $8(47.1)$ & $27(54)$ & \multirow[t]{2}{*}{$0.621^{\mathrm{a}}$} \\
\hline$<150$ & & $9(52.9)$ & $23(46)$ & \\
\hline \multicolumn{5}{|l|}{ Hypertension } \\
\hline Yes & $n(\%)$ & $17(48.6)$ & $46(54.1)$ & \multirow[t]{2}{*}{$0.580^{\mathrm{a}}$} \\
\hline No & & $18(51.4)$ & $39(45.9)$ & \\
\hline
\end{tabular}

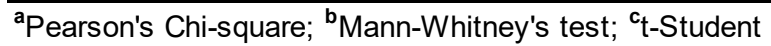

Abbreviations: FSGS: Focal segmental glomerulosclerosis; SD: Standard deviation; $\mathbf{n}$ : Sample number

who belonged to the fFSGS group were resistant to the steroids $(100 \%)$, while the sFSGS behaved differently $(59.7 \%)(p=0.001)$.

\section{Discussion}

FSGS is a common histological manifestation shared by possibly different glomerular diseases that may not be distinguished by routinely histopathological analysis, which represents an obstacle in the adequate treatment of the disease.

Our study presents evidence that sFSGS and fFSGS share some clinical characteristics but behave differently with regards to the treatment with steroids and immunosuppressive drugs, which may have an impact on clinical daily practice. In the present study we evaluated 124 patients with FSGS searching for eventual clinical and laboratorial features that could contribute to the differential diagnosis and management of familial and sporadic forms of FSGS. It is worth to mention that this study was developed in a service that assists patients who are $\geq 12$ years-old.

Our results confirm that fFSGS and sFSGS are similar with regards to gender, ethnicity, age at disease onset, baseline serum creatinine (mean of 1.20 vs. $1.31 \mathrm{mg} /$ $\mathrm{dL}$ ), hypertension, hematuria, hypercholesterolemia and hypertriglyceridemia.

We observed that in sFSGS (vs. fFSGS), 24-hour proteinuria had a trend to be higher than $3.0 \mathrm{~g} / 24 \mathrm{~h}$ $(p=0.080)$ associated with lower levels of serum albumin $(p=0.046)$, which shows that the sporadic form possibly present more often with nephrotic syndrome, while the familial form tends to have non-nephrotic proteinuria. 
Such findings may not impact on the differential diagnosis between sFSGS and fFSGS when considered a particular individual and not a group, but they can guide the treatment and help the physicians to choose whether using renoprotective drugs only for treating fFSGS.

Remission was observed only in part of the patients with sFSGS (60\%) after steroid therapy, as expected. However, $100 \%$ of the fFSGS patients were resistant to steroids $(\mathrm{p}=0.001)$. It is of note that immunosuppressive therapy is usually avoided in fFSGS, but consensual guidelines still lack to approach this condition ${ }^{16}$, and many patients with fFSGS are still submitted to steroid therapy.

In the present study, some laboratory characteristics were statistically different between fFSGS and sFSGS proteinuria and serum albumin levels and have clinical significance. We consider such parameters as additional features to distinguish between these two forms of FSGS while assessing the patient. Certainly, these findings contribute to distinguishing these two forms of FSGS but they are not enough. In addition, their patterns of proteinuria and degree of hypoalbuminemia may have an impact on the rate of progression to end-stage kidney disease of each form of FSGS, considering the importance of persistent nephrotic syndrome (in particular high levels of proteinuria and low levels of serum albumin in the present study) on prognosis.

Among the limitations of the present study, we highlight that the diagnosis of FSGS may be challenging, and it may be difficult to distinguish FSGS from collagen IV diseases based on histological evaluation, particularly if electron microscopy is not available.

It is of note that the prevalence of genetic FSGS is markedly higher in children than in adolescents and adults, and the conclusions of the present study are not applicable for children.

For the age range here studied although the clinical characteristics of FSGS as familial or sporadic can suggest the treatment, genetic studies are also recommended in some cases. Previously our group showed that the genetic analysis is little helpful among adults who likely present the sporadic form of $\mathrm{FSGS}^{17}$, regardless of the fact that associated pathogenic mutations can occur in fFSGS as well as in sFSGS, in children, adolescents and adults.

Genetic diagnosis in adult-onset FSGS can have farreaching consequences not only for the patient but also for his/her family. Regardless of the role of the available gene mutation panels of FSGS, a family history of kidney disease may help to differentiate between both forms of FSGS. In fact, a careful medical history may raise a suspicion for fFSGS, indicating an active search for other affected individuals in fFSGS, even among relatives that are still asymptomatic, as well as the best disease management. Then a pedigree may help to identify the inheritance pattern and so suggest the more appropriate medical approach.

\section{Conclusions}

Pointed distinguishing features of sFSGS (higher proteinuria and lower serum albumin, steroid responsiveness when present) and fFSGS (family history of glomerular and/or chronic kidney disease) can contribute to disease management, including the treatment approach. It is of note that a thorough disease history will be the first clue for such differential diagnosis.

Genetic study can be recommended after considering the prognosis and the therapeutic implications of finding a pathogenic variant both in the patients or their relatives. This information may guide genetic counseling especially when a relative intend to donate a kidney for transplantation in case of fFSGS in addition to the screening for renal disease.

\section{Abbreviations}

ACTN4: Alpha-actinin-4

CD2AP: CD2-associated protein

FSGS: Focal segmental glomerulosclerosis

fFSGS: Familial focal segmental glomerulosclerosis

INF2: Inverted formin 2

NPHS2: Podocin

sFSGS: Sporadic focal segmental glomerulosclerosis

TRPC6: Short transient receptor potential channel 6

\section{Authors' contributions}

MGP, MTP and DEF collected data and performed the analyses under the supervision of GM-K. All the authors had access to the data and worked on the final version of this manuscript.

\section{Competing interests}

The authors declare no competing interests.

\section{Acknowledgements and Funding}

This study was supported by a CNPq (National Council for Research and Development) grant CNPq/MS/SCTIE/ DECIT - nr. 36/2014. MGP and MTP are received PNPDCAPES (National Postdoctoral Program/ Coordination of Improvement of Higher Education Personnel) grant process nr. 1668138.

\section{References}

1. Faubert PF, Porush JG. Familial focal segmental glomerulosclerosis: Nine cases in four families and review of the literature. Am J Kidney Dis. 1997; 30(2): 265-270.

2. Conlon PJ, Lynn K, Winn MP, et al. Spectrum of disease in familial focal and segmental glomerulosclerosis. Kidney Int. 1999; 56(5): 18631871. 
3. Frishberg Y, Rinat C, Megged O, et al. Mutations in NPHS2 encoding podocin are a prevalent cause of steroid-resistant nephrotic syndrome among Israeli-Arab children. J Am Soc Nephrol. 2002; 13(2): 400-405.

4. Feltran LS, Varela P, Silva ED, et al. Targeted Next-Generation Sequencing in Brazilian Children With Nephrotic Syndrome Submitted to Renal Transplant. Transplantation. 2017; 101(12): 2905-2912.

5. De Vriese AS, Sethi S, Nath KA, et al. Differentiating Primary, Genetic, and Secondary FSGS in Adults: A Clinicopathologic Approach. JASN. Published online January 10, 2018: ASN.2017090958. doi:10.1681/ ASN.2017090958

6. Caridi G, Bertelli R, Carrea A, et al. Prevalence, genetics, and clinical features of patients carrying podocin mutations in steroid-resistant nonfamilial focal segmental glomerulosclerosis. J Am Soc Nephrol. 2001; 12(12): 2742-2746.

7. Caridi G, Bertelli R, Di Duca M, et al. Broadening the Spectrum of Diseases Related to Podocin Mutations. J Am Soc Nephrol. 2003; 14(5): 1278-1286.

8. Riguetti MTP, Varela P, Fernandes DE, et al. Familial Focal Segmental Glomerulosclerosis With Late-Onset Presentation and R229Q/ R291W Podocin Mutations. Front Genet. 2020; 11: 533373.

9. Sanchez-Ares M, Garcia-Vidal M, Antucho E-E, et al. A novel mutation, outside of the candidate region for diagnosis, in the inverted formin 2 gene can cause focal segmental glomerulosclerosis. Kidney Int. 2013; 83(1): 153-159.

10. Zhang Q, Ma J, Xie J, et al. Screening of ACTN4 and TRPC6 Mutations in a Chinese Cohort of Patients with Adult-Onset Familial Focal Segmental Glomerulosclerosis. Contrib Nephrol. 2013; 181: 91-100.

11. Barua M, Brown EJ, Charoonratana VT, et al. Mutations in the INF2 gene account for a significant proportion of familial but not sporadic focal and segmental glomerulosclerosis. Kidney Int. 2013; 83(2): 316322.

12. Kang HG, Ha I-S, Cheong HI. Recurrence and Treatment after Renal Transplantation in Children with FSGS. BioMed Res Int. 2016; 2016 : 1-7.

13. Faul C, Donnelly M, Merscher-Gomez S, et al. The actin cytoskeleton of kidney podocytes is a direct target of the antiproteinuric effect of cyclosporine A. Nat Med. 2008; 14(9): 931-938.

14. Machuca E, Hummel A, Nevo F, et al. Clinical and epidemiological assessment of steroid-resistant nephrotic syndrome associated with the NPHS2 R229Q variant. Kidney Int. 2009; 75(7): 727-735.

15. Büscher AK, Kranz B, Büscher R, et al. Immunosuppression and renal outcome in congenital and pediatric steroid-resistant nephrotic syndrome. Clin J Am Soc Nephrol. 2010; 5(11): 2075-2084.

16. Yorgin PD, Belson A, Higgins J, et al. Pulse methylprednisolone cyclosporine, and ACE inhibitor therapy decreases proteinuria in two siblings with familial focal segmental glomerulosclerosis. Am J Kidney Dis. 2001; 37(6): e44.1-e44.6.

17. Monteiro EJ, Pereira AC, Pereira AB, et al. NPHS2 mutations in adult patients with primary focal segmental glomerulosclerosis. J Nephrol. 2006; 19(3): 366-371. 